Perceptual and Motor Skills, 2004, 98, 1039-1048. C Perceptual and Motor Skills 2004

\title{
IMPROVING ACCURACY OF VERACITY JUDGMENT THROUGH CUE TRAINING ${ }^{1}$
}

\author{
MARIA SANTARCANGELO, ROBERT A. CRIBBIE \\ AMY S. EBESU HUBBARD \\ York University \\ University of Hawaii at Manoa
}

\begin{abstract}
Summary.-The ability to make judgments of veracity was investigated to see if training individuals on visual, vocal, or verbal content cues of deception would increase their ability to judge whether a message was truthful. The overall rate of accuracy of judging veracity was significantly greater for subjects trained on verbal content cues. More specifically, for detecting truthful messages, subjects trained on verbal content cues had significantly greater accuracy than subjects who received no cue training, whereas for detecting deceptive messages, there were no significant differences in accuracy among conditions.
\end{abstract}

Within daily interactions people often come across instances in which they must make judgments of veracity, i.e., evaluate whether someone is lying or telling the truth. Previous studies examining the rate of accuracy of human lie detection have shown the average accuracy barely exceeds chance expectations (Hocking \& Leathers, 1980; DeTurck \& Miller, 1985; Kohnken, 1987; DePaulo, Tornqvist, \& Cooper, 2002). Even when the individuals who were detecting the deception were friends of the person communicating the message, the rates of accuracy were not significantly greater than chance (Anderson, DePaulo, \& Ansfield, 2002). Stiff and Miller (1986) found that, when making judgments about the veracity of a message, individuals relied on a number of visual, vocal, and verbal cues unrelated to the actual veracity of the message. Research examining the cues used by individuals to make judgments of veracity has indicated little correspondence between the cues individuals perceive to be related to deception and the actual cues related to deception (DePaulo, Rosenthal, Rosenkrantz, \& Green, 1982; DeTurck \& Miller, 1985; Fiedler \& Walka, 1993; Anderson, DePaulo, Ansfield, Tickle, \& Green, 1999; DePaulo, Tornqvist, \& Cooper, 2002). Zuckerman, Koestner, and Driver (1981) and Zuckerman, Koestner, and Colella (1985) investigated the beliefs about cues associated with deception and found that there are common stereotypes people hold about cues for deception and that these stereotyped cues were minimally correlated with actual cues for deception.

Beliefs about deception cues are often attributed to arousal theories of deception. Detecting deception is based on the assumption that a person

\footnotetext{
${ }^{1}$ Address correspondence to Robert A. Cribbie, Department of Psychology, York University, Toronto, ON Canada M3J 1P3 or e-mail (cribbie@yorku.ca).
} 
who is being deceptive will experience increased physiological arousal compared to a person who is telling the truth. This arousal is an indicator of the guilty feelings, anxiety, embarrassment, or nervousness associated with lying. It is also assumed that arousal is an indicator of stress associated with cognitive attempts to construct believable messages or efforts to avoid mistakes (Hocking \& Leathers, 1980; DeTurck \& Miller, 1985; Fiedler \& Walka, 1993). Detecting deception is made possible through the display of physiological arousal. Deception-induced arousal can be detected in physical, vocal, and verbal displays. However, a person who is being deceptive will deliberately try to control their behavioral displays to avoid suspicion (Hocking \& Leathers, 1980; Zuckerman, et al., 1981). According to Kraut (1978), "one should believe most in those aspects of a person's performance that the person is least able to deliberately and consciously control" (p. 381). Research has supported this statement and has found that there are behavioral displays unique to deception-induced arousal. DeTurck and Miller (1985) investigated the behavioral differences between people being deceptive and aroused truth-tellers and found that adaptors (such as scratching, stroking), hand gestures, speech errors, pauses, response latency, and message duration reliably distinguished people being deceptive from unaroused truth-tellers and also from aroused truth-tellers.

Training individuals on the behavioral cues related to deception-induced arousal has increased the rate of accuracy of detecting deception (DeTurck \& Miller, 1990; Fiedler \& Walka, 1993). Training improved the ability to detect deception in those who were high self-monitors and who had rehearsed their lies. These are two groups whose deception is difficult to detect (DeTurck \& Miller, 1990). DePaulo, Lassiter, and Stone (1982) found that subjects merely instructed to pay attention to tone of voice or words were more accurate at detecting deception than those who were told to pay attention to visual cues. On the other hand, Kohnken (1987) trained police officers to detect deception and found subjects who were trained were no more accurate than subjects in the control group who received no training. Kohnken concluded that cue training may have led to information-processing overload. Subjects in this study had to learn an extensive set of behavioral cues which hindered their detection of deception. By using fewer cues, observers may more fully utilize the cue information and make more accurate judgments.

Research on deception has often focused on examining the behaviors associated with deception communication. Results of these studies indicate that there are consistent verbal and nonverbal cues related to actual deception. For the purpose of this study, these cues have been categorized into verbal content cues, vocal cues, and visual cues. 


\section{Verbal Content Cues}

Little research has focused on the verbal content cues related to deception. This is due to the belief that verbal content can be monitored and controlled by the speaker (Kraut, 1978; DePaulo, Rosenthal, Rosenkrantz, \& Green, 1982). However, research on verbal content cues has indicated that cues within the speech content of people being deceptive (and those telling the truth) can actually be more reliable in detection of deception than nonverbal cues. DePaulo, Rosenthal, Rosenkrantz, and Green (1982) reported that speech can reliably indicate whether deception is occurring and that perceivers are often strongly influenced by speech in their judgments about deceit. In a study comparing verbal cues, vocal cues, and visual cues, only one verbal cue (verbal content) was related to actual deception (Stiff \& Miller, 1986). Kraut's study (1978) on verbal content cues and nonverbal cues identified six of nine behavioral cues as actually related to deception, of which four were verbal content cues. The four verbal content cues related to veracity of a message are plausibility, concreteness, consistency, and clarity. Research has found that people being deceptive give less plausible, less concrete, less consistent, and less clear responses.

Vocal Cues

Vocal cues are defined as the cues within speech, excluding meaning. Research has found that twice as many vocal behaviors as opposed to nonverbal behaviors distinguished people being deceptive from those telling the truth and has consistently shown vocal behaviors to be more reliable indices of deception than nonverbal behaviors (DeTurck \& Miller, 1985). These include response duration, pauses, speech errors, and response latency. These four cues have been consistently related to actual deception. People being deceptive produced shorter responses, more pauses during speech, more speech errors, and longer response latency (DeTurck \& Miller, 1985; Stiff \& Miller, 1986; Kohnken, 1987).

\section{Visual Cues}

Visual cues have received the most attention in research and yet have yielded the most inconsistent findings. Several studies yielded increases in the number of visual cues during deception, and in other studies decreases in the number of visual cues in deception have been noted. The increases in visual cues have been linked to the hypothesis that deception elicits arousal, for example, increased nervousness results in more hand gestures and foot movements (Vrij, 1995). The decreases in visual cues can be explained by people being more deceptive believing that movements give their lies away so they tend to avoid movements not strictly essential. This control results in an unusual rigidity and inhibition (Hocking \& Leathers, 1980; Vrij, 1995). Research has also found that observers largely rely on visual cues when mak- 
ing judgments of deception, and possibly these visual cues may serve as distractors in the process of detecting deception (Stiff \& Miller, 1986; Stiff, Miller, Sleight, Mongeau, Rogan, \& Garlick, 1989). The visual cues consistently related to actual deception are self-adaptors (scratching, grooming, stroking the face, etc.), hand gestures, foot and leg movements, and postural shifts. A person who is being deceptive engages in more of these behaviors.

The purpose of this study is to investigate whether providing short-term training to individuals on behavioral cues related to deception will increase their correct judgment of veracity compared to individuals who received no training. This study investigated the behavioral cues related to judgments of veracity to assess which set of cues is most effective in improving judgment of veracity. It was hypothesized that participants who received cue training would have a higher proportion of correct judgments of veracity than those participants who received no cue training. For participants who received cue training, it was hypothesized that participants trained on verbal content cues would have the highest accuracy of judgments of veracity.

\section{Method}

\section{Participants}

Ninety-seven first-year undergraduate psychology students volunteered to participate in the study and received course credit for their participation. There were 16 men and 81 women, ranging in age from 18 to 43 years $(M=$ 20.8).

\section{Materials}

A stimulus videotape of interviews with 60 subjects, 45 minutes long, was adapted from videotapes utilized by Ebesu and Miller (1994) who had individuals produce one of four different messages within different scenarios: (1) lie, (2) misdirection, (3) implying the truth, and (4) telling the truth. Subjects were videotaped so that their entire bodies were seen easily. The truth and lie message conditions were extracted from these videotapes with permission for use in this study. Specifically, 30 subjects giving truth messages and 30 different subjects giving lie messages were randomly selected and ordered, producing a 45 -min. videotape of 60 subjects, each one telling either the truth or a lie.

Handouts were created for the three experimental conditions and the control condition. For the experimental conditions, the handout consisted of a training sheet and a response sheet. The training sheets for the verbal content, visual, and vocal cues consisted of four cues, each defined and explained with examples. Subjects in the verbal content cue condition were trained to be sensitive to the plausibility, concreteness, consistency, and clarity of the statements. Subjects in the visual cue condition were trained to be 
sensitive to the presence of self-adaptors (such as rubbing your face), hand gestures, foot/leg movements, and postural shifts. Lastly, subjects in the vocal cues condition were trained to be sensitive to response duration, the presence of pauses, speech errors, and response latency, i.e., hesitation before speaking.

\section{Procedure}

Participants were randomly assigned to one of the four conditions. Participants were seated before a television screen on which the stimulus videotape was presented. Participants in the cue training conditions were given the corresponding training sheets to study and, in addition, the experimenter read each behavioral cue with associated definitions and examples. Participants were provided an opportunity to ask for clarification on any of the behavioral cues. Participants in the control condition received no written or oral information on behavioral cues related to deception. Participants in both experimental and control conditions were instructed that they would be shown a $45-\mathrm{min}$. videotape of 60 individuals, each of whom was either telling the truth or a lie. After viewing each individual, the participants were instructed to indicate on their response sheet whether they judged the individual to be telling the truth or a lie.

\section{Results}

Means and standard deviations for each training condition across each type of message are displayed in Table 1. An alpha level of .05 was adopted for all tests of significance. Distribution shapes were approximately normal in form for each message type within each condition. All tests of mean differences were performed with Welch's heteroscedastic two-sample and omnibus statistics, respectively (1938, 1951; cf. Keselman, Huberty, Lix, Olejnik, Cribbie, Donahue, Kowalchuk, Lowman, Petoskey, Keselman, \& Levin, 1998). Where necessary, the Games-Howell heteroscedastic pairwise multiple comparison procedure (Games \& Howell, 1976) was utilized (in essence,

TABLE 1

Mean Accuracy and Standard Deviations For Training Conditions by Correct Judgment of All, Truthful, and Deceptive Messages

\begin{tabular}{|c|c|c|c|c|c|c|c|}
\hline \multirow[t]{3}{*}{ Condition } & \multirow[t]{3}{*}{$n$} & \multicolumn{6}{|c|}{ Correct Judgment of Message } \\
\hline & & \multicolumn{2}{|c|}{ All 60} & \multicolumn{2}{|c|}{30 True } & \multicolumn{2}{|c|}{$30 \mathrm{Lie}$} \\
\hline & & $M$ & $S D$ & $M$ & $S D$ & $M$ & $S D$ \\
\hline No Cue Training & 30 & 38.8 & 5.5 & 21.0 & 4.9 & 17.6 & 2.7 \\
\hline All Cue Training & 67 & 41.3 & 4.8 & 23.6 & 4.1 & 17.7 & 3.6 \\
\hline Visual Cues & 21 & 40.4 & 4.6 & 23.7 & 4.4 & 16.7 & 3.6 \\
\hline Vocal Cues & 20 & 40.4 & 5.9 & 22.8 & 4.9 & 17.6 & 4.2 \\
\hline Verbal Content Cues & 26 & 42.9 & 3.7 & 24.3 & 3.1 & 18.6 & 2.8 \\
\hline
\end{tabular}


Tukey's honestly significant difference familywise error control with the Welch heteroscedastic statistic). In addition, recent research has reported that model-testing procedures, which eliminate intransitivity and provide a more logical method for evaluating pairwise mean differences, can also be much more likely to detect the true underlying population mean configuration than traditional pairwise multiple comparison procedures (e.g., Dayton, 1998, 2003; Cribbie, 2003; Cribbie \& Keselman, 2003). Thus, the modeltesting procedure proposed by Dayton (1998) was also utilized in evaluating differences in the means of the conditions. With this procedure the model with the lowest Akaike Information Criteria value (see Dayton, 1998, 2003) was retained as the most probable population mean configuration (assuming the omnibus test of mean difference was statistically significant, see Cribbie \& Keselman, 2003). In essence, the Dayton model-testing procedure conducts pairwise comparisons of the means but does so in a way that is transitive, i.e., a condition/group cannot be drawn from two distinct populations, and wholistic, i.e., the results are interpretable within the framework of a population model rather than within isolated two-group comparisons.

To assess whether training individuals on behavioral cues related to deception would improve their correct judgment of the truthfulness of a message, a two-sample Welch test was conducted to compare the overall detection by participants who received cue training with that of participants who received no cue training. There was a significant mean difference in accuracy between participants who received cue training and those who did not $\left(t_{49.87}\right.$ $=2.13, p=.04, \eta^{2}=.09$ ), with the former having a higher proportion of correct judgments of veracity than the latter. The significant mean difference between the experimental and control groups indicates that, as predicted, training individuals on behavioral cues related to deception improved their overall detection. More specifically, there was a significant mean difference in accuracy between participants who received cue training and those who did not receive cue training for detecting when subjects were telling the truth $\left(t_{48.31}=2.63, p=.01, \eta^{2}=.13\right)$, showing those who received cue training had a higher proportion of correct judgments. However, there was no significant difference between participants who received cue training and those who did not receive cue training for detecting when subjects were not telling the truth $\left(t_{71.36}=0.18\right.$, ns, $\left.\eta^{2}<.01\right)$.

To examine the relative importance of each set of behavioral cues in judging the message's veracity, an omnibus Welch test was conducted to compare the overall detection of participants trained on verbal content cues, visual cues, and vocal cues with that of individuals who received no cue training. There was a significant mean difference in accuracy between these two groups of participants $\left(F_{3,48.01}=3.77, p=.016, \eta^{2}=.09\right)$. Pairwise comparisons indicated that the participants trained on verbal content cues had a sig- 
nificantly higher proportion of correct judgments of veracity than the participants who received no cue training. Dayton's model-testing procedure (1998) indicated that subjects who were trained to be sensitive to verbal content cues were drawn from a population distinct from those who had no training or who were trained to be sensitive to vocal or visual cues (see Table 2).

TABLE 2

Daytonss Model Testing Procedure (1998): Results For Eight Potential Transitive Population Mean Configurations

\begin{tabular}{|c|c|c|}
\hline \multirow[t]{2}{*}{ Mean Population Configuration* } & \multicolumn{2}{|c|}{ Akaike Information Criteria } \\
\hline & Overall & Truth \\
\hline [Control, Vocal, Visual, Verbal Content] & 595.29 & 570.57 \\
\hline [Control] [Vocal, Visual, Verbal Content] & 592.01 & $564.43 \dagger$ \\
\hline [Control, Vocal] [Visual, Verbal Content] & 592.00 & 565.34 \\
\hline [Control, Vocal] [Visual] [Verbal Content] & 590.96 & 567.14 \\
\hline [Control, Vocal, Visual] [Verbal Content] & $589.49 *$ & 568.54 \\
\hline [Control] [Vocal] [Visual, Verbal Content] & 592.83 & 565.17 \\
\hline [Control] [Vocal, Visual] [Verbal Content] & 589.79 & 565.49 \\
\hline [Control] [Vocal] [Visual] [Verbal Content] & 591.79 & 566.97 \\
\hline
\end{tabular}

*Conditions enclosed within brackets represent distinct populations. $†$ The model with the lowest Akaike Information Criteria value is retained as the most likely mean population configuration.

The effect of cue training on detection was further investigated by examining the detection of message type. To assess whether training individuals on cues related to deception would improve their detection of truthful messages, an omnibus Welch test was conducted, comparing the proportion of correctly judged truthful messages of individuals in each training condition. There was a significant mean difference in accuracy $\left(F_{3,47.58}=3.21, p=\right.$ $.031, \eta^{2}=.09$ ), and pairwise comparisons showed participants who received training on verbal content cues had a significantly higher proportion of correct truth judgments than those who received no cue training. Dayton's model-testing procedure (1998) indicated subjects who were trained to be sensitive to any of the cues related to deception were drawn from a population distinct from those who had no training in the cues related to deception (see Table 2).

Whether training individuals on cues related to deception would improve their detection of deceptive messages was tested with an omnibus Welch test comparing the proportion of correctly judged deceptive messages in each condition. There was no significant mean difference in accuracy in detection of deceptive messages between participants in the four training conditions $\left(F_{3,46.60}=1.42\right.$, ns, $\left.\eta^{2}=.04\right)$. 


\section{Discussion}

The present study investigated judgments of the truth or falsehood of a message. The primary purpose was to investigate the effect of short-term cue training on individuals' judgments of this veracity. It was hypothesized that cue training would increase detection accuracy. Participants who received cue training were more accurate at judging veracity than the participants in the control condition, who received no cue training. Participants who received information on cues associated with actual deception (and how to use these cues effectively in making judgments) were significantly better than the control group on the overall detection of truthful and deceptive messages. It was also hypothesized that participants trained on verbal content cues would be more accurate in judging veracity of a message than would the other cue sets and control condition. The results, both in terms of traditional pairwise comparison analyses and the preferable model-testing procedure of Dayton (1998), confirmed that verbal content cues were the most effective training cues. Short-term training in the detection of plausibility, concreteness, consistency, and clarity cues significantly improved correct judgments of veracity relative to judgments of subjects in the control condition. This finding is consistent with results of Stiff and Miller (1986), Kraut (1978), and others, as well as with the results of a large meta-analysis by DePaulo, Lindsay, Malone, Muhlenbruck, Charlton, and Cooper (2003), wherein verbal content cues (such as the plausibility, logical structure, discrepancy/ambivalency, or amount of detail), unlike most other cues investigated in the literature on deception, significantly increased accuracy of judgments of veracity. These findings support the use of verbal content training for individuals, e.g., police officers, who are routinely required to differentiate between truthful and untruthful messages, although it is important to acknowledge that accuracy rates are still below $70 \%$, and more research should be done into factors that help improve overall accuracy; see DePaulo, et al. 2003 for an extensive discussion on this topic.

In detection of truthful statements, participants trained on verbal content cues had a significantly higher accuracy rate than the control condition, and Dayton's model-testing procedure indicated all training conditions produced better performance than that of the control condition. In detecting untruthful statements, no significant differences in accuracy were found among the conditions as rates of accuracy were only minimally above chance performance. The poor accuracy rate for detecting deceptive messages is consistent with previous research showing deception detection is no greater than chance expectation (Hocking \& Leathers, 1980; DeTurck \& Miller, 1985; Kohnken, 1987). This could be a result of the truthfulness bias, that incorrectly judging a deceptive statement as truthful is more likely than judging a truth statement as deceptive (Kohnken, 1987; Anderson, et al., 2002). 
The participants who did not receive cue training illustrate that humans' judgments of veracity do not exceed chance expectations. These individuals were significantly less accurate at detecting truthful statements and at making overall judgments of veracity.

One weakness of this study was that subjects were judging the truthfulness of individuals who were sanctioned to lie or tell the truth. The individuals who were presented in the videotape may not have been displaying the physiological arousal associated with deception because they were enacting imaginary scenarios that led to no serious consequences of their deception. These individuals may not have been experiencing the guilt and anxiety typically associated with deception that would have otherwise possibly been evident. This could also explain why the deceptive messages were so difficult to detect by the training conditions, as there may not have been significant displays of cues for subjects to make accurate judgments. However, another way to interpret this is that, even though the cues for detecting deception were not highly evident, the subjects trained even briefly on detecting verbal content cues were still more likely to make accurate judgments of veracity. In this manner, it may be possible that subjects who are more extensively trained to detect verbal content cues may be even more accurate in detecting the truthfulness of a message, relative to subjects with no cue training or with training in identifying other types of behavioral cues, when the individual whom they judged to be lying or telling the truth is, in reality, actually telling the truth or lying and not a confederate of the experiment.

\section{REFERENCES}

Anderson, D. E., DePaulo, B. M., \& Ansfield, M. E. (2002) The development of deception detection skill: a longitudinal study of same-sex friends. Personality and Social Psychology Bulletin, 28, 536-545.

Anderson, D. E., DePaulo, B. M., Ansfield, M. E., Tickle, J. J., \& Green, E. (1999) Beliefs about cues to deception: mindless stereotypes or untapped wisdom? Journal of Nonverbal Bebavior, 23, 67-89.

Cribbie, R. A. (2003) Pairwise multiple comparisons: new yardsticks, new results. Journal of Experimental Education, 71, 251-265.

Cribbie, R. A., \& Keselman, H. J. (2003) Pairwise multiple comparisons: a model comparison approach versus stepwise procedures. British Journal of Mathematical and Statistical Psy. chology, 56, 167-182.

Dayton, C. M. (1998) Information criteria for the paired-comparisons problem. The American Statistician, 52, 144-151.

DaYton, C. M. (2003) Information criteria for the pairwise comparisons. Psychological Methods, 8, 61-71.

DePaulo, B. M., Lassiter, G. D., \& Stone, J. I. (1982) Attentional determinants of success at detecting deception and truth. Personality and Social Psycbology Bulletin, 8, 273-279.

Depaulo, B. M., Lindsay, J. J., Malone, B. E., Muhlenbruck, L., Charlton, K., \& Cooper, H. (2003) Cues to deception. Psychological Bulletin, 129, 74-118.

DePaulo, B. M., Rosenthal, R., Rosenkrantz, J., \& Green, C. R. (1982) Actual and perceived cues to deception: a closer look at speech. Basic and Applied Social Psychology, 3, 291312.

DePaulo, B. M., Tornquist, J. S., \& Cooper, H. (2002) Accuracy at detecting deception: a meta-analysis of modality effects. (Unpublished manuscript, Univer. of Virginia) 
DeTurck, M. A., \& Miller, G. R. (1985) Deception and arousal: isolating the behavioral correlates of deception. Human Communication Research, 12, 181-201.

DeTurck, M. A., \& Miller, G. R. (1990) Training observers to detect deception: effects of self-monitoring and rehearsal. Human Communication Research, 16, 603-620.

Ebesu, A. S., \& Miller, M. D. (1994) Verbal and nonverbal behaviors as a function of deception type. Journal of Language and Social Psychology, 13, 418-442.

Fiedler, K., \& WaLKa, I. (1993) Training lie detectors to use nonverbal cues instead of global heuristics. Human Communication Research, 20, 199-223.

Games, P. A., \& Howell, J. F. (1976) Pairwise multiple comparison procedures with unequal $n$ 's and/or variances. Journal of Educational Statistics, 1, 113-125.

Hocking, J. E., \& Leathers, D. G. (1980) Nonverbal indicators of deception: a new theoretical perspective. Communication Monographs, 47, 119-131.

Keselman, H. J., Huberty, C. J., Lix, L. M., Olejnik, S., Cribbie, R., Donahue, B., Kowalchuk, R. K., Lowman, L. L., Petoskey, M. D., Keselman, J. C., \& Levin, J. R. (1998) Statistical practices of educational researchers: an analysis of their ANOVA, MANOVA, and ANCOVA analyses. Review of Educational Research, 68, 350-386.

KohnKen, G. (1987) Training police officers to detect deceptive eyewitness statements: does it work? Social Bebavior, 2, 1-17.

KRAUT, R. E. (1978) Verbal and nonverbal cues in the perception of lying. Journal of Personality and Social Psychology, 36, 380-391.

Stipf, J. B., \& Miller, G. R. (1986) "Come to think of it": interrogative probes, deceptive communication, and deception detection. Human Communication Research, 12, 339-357.

Stiff, J. B., Miller, G. R., Sleight, C., Mongeau, P., Rogan, R., \& Garlick, R. (1989) Explanations for visual cue primacy in judgments of honesty and deceit. Journal of Personality and Social Psychology, 56, 555-564.

VRI, A. (1995) Behavioral correlates of deception in a simulated police interview. The Journal of Psychology, 129, 15-28.

WELCH, B. L. (1938) The significance of the difference between two means when population variances are unequal. Biometrika, 29, 350-362.

WeLCH, B. L. (1951) On the comparison of several mean values: an alternative approach. Biometrika, 38, 330-336.

Zuckerman, M., Koestner, R., \& Colella, M. J. (1985) Learning to detect deception from three communication channels. Journal of Nonverbal Bebavior, 9, 188-194.

Zuckerman, M., Koestner, R., \& Driver, R. (1981) Beliefs about cues associated with deception. Journal of Nonverbal Bebavior, 6, 105-114. 